sources: "Most of the leading scientists of eighteenth-century Europe", he says in citing D. Cardwell, "were instrumentmakers by profession". But Cardwell had said, and only in parentheses, that "Some of the leading scientists of the century were instrument-makers by profession" (emphases mine). Was Joseph Priestley an instrument-maker? What about John Dalton and Joseph Black?

To be sure, Kumar the historian of science has no access to primary sources at all; contrast it with Kumar the historian of colonialism. It is embarrassing to express firm views on, for example, the history of Indian astronomy without looking at a single Sanskrit text, not to mention Arabic and Persian writings. And even thesecondary sources here are highly unreliable. The case of the astronomerruler Maharaja Jai Singh is one glaring instance. Kumar says, on the authority of a poor secondary reference, that the Maharaja had accepted the Copernican model; this is simply wrong. Kumar would be well advised to consult the researches of historians such as K. P. Shukla, V. N. Sharma, S. M. R. Ansari and David Pingree. To measure the worth of the Indian scientific tradition in terms of its proximity to modern science is to betray an unhappy lack of understanding of the scientific spirit and scientific enterprise.

The author also raises the vexed question of science and ideology, and contradicts himself. On the one hand, he quotes with approval the views of al-Afghânî and Sir Syed Ahmed Khan (there is no 'European' science and 'Islamic' science, science is science; science is secular, universal, ideology-free, and so on); on the other hand, he himself talks about "colonial science" - in fact he goes as far as to say: "Colonial science... had an ideology"; in other words, science is not utterly ideology-free. But if this is the case, why does he disapprove of the ideological concerns of the nineteenth-century Indian scientists such as P. N. Bose, P. C. Ray and R. Trivedi who spoke of Hindu culture, civilization, cosmology and metaphysics? Why do they betray "streaks of revivalism"? It seems to me that these people understood the nature of science better than Sir Syed did; and they had a more powerful intellect than their contemporary Chattopadhyaya who declared so naively - and with such a ringing apologetic tone - that the system of Âryabhata is identical to that of Copernicus; this is absurd.

If Science and the Raj is about colonialism it is welcome; if it makes any claims to being a history of science, it ought be ignored.

S. Nomanul Haq is in the Department of Religious Studies, Brown University, Providence, Rhode Island 02912, USA.

\section{Interrogation and interpretation}

Donald J. DePaolo

Introduction to Geochemical Modeling. By Francis Albarède. Cambridge University Press: 1995. Pp. 543. £80, $\$ 100$ (hbk); $£ 29.95, \$ 39.95$ (pbk).

THE focus of most geochemistry is the interrogation of nature with ever more penetrating measurements - higher precision, better spatial resolution, greater sensitivity. The Holy Grail of geochemistry could be said to be the 'triumphant measurement', the implications of which are both profound and patently obvious, if not quite model-independent. Who is to deny that the Solar System is 4.55 billion years old, a statement so elegantly made by leadisotope measurements?

But most geochemical data, especially those that prove to be indispensable for environmental and geological problems, require involved interpretation regardless of the sophistication of the measurements. The ultimate value of geochemical data, no matter how beautiful they appear to the creator and the connoisseur, is no more and no less than as a constraint on a model, and the poorer the model, the less valuable the data.

Francis Albarède has produced an impressive and much needed contribution to the continuing transformation of geochemistry into an explicit model-based science. Most of the essential mathematics of geochemistry is presented in a clear and readable fashion. The special value of the book lies in its relative completeness, in both the breadth of topics and approaches covered and the step-by-step working of relevant problems.

This is an indispensable handbook for the research geochemist, and could serve as a text for a graduate or advanced undergraduate geochemistry course. Graduate students will find the book invaluable for access to the geochemical literature, as well as for dealing with their own data. Albarède presents mathematics admirably by illustrating the applications to common geochemical problems while revealing his personal vision of quantitative geochemistry and providing a useful introduction to the attendant terminology.

Donald J. DePaolo is in the Department of Geology and Geophysics, University of California, Berkeley, California 947204767, USA.

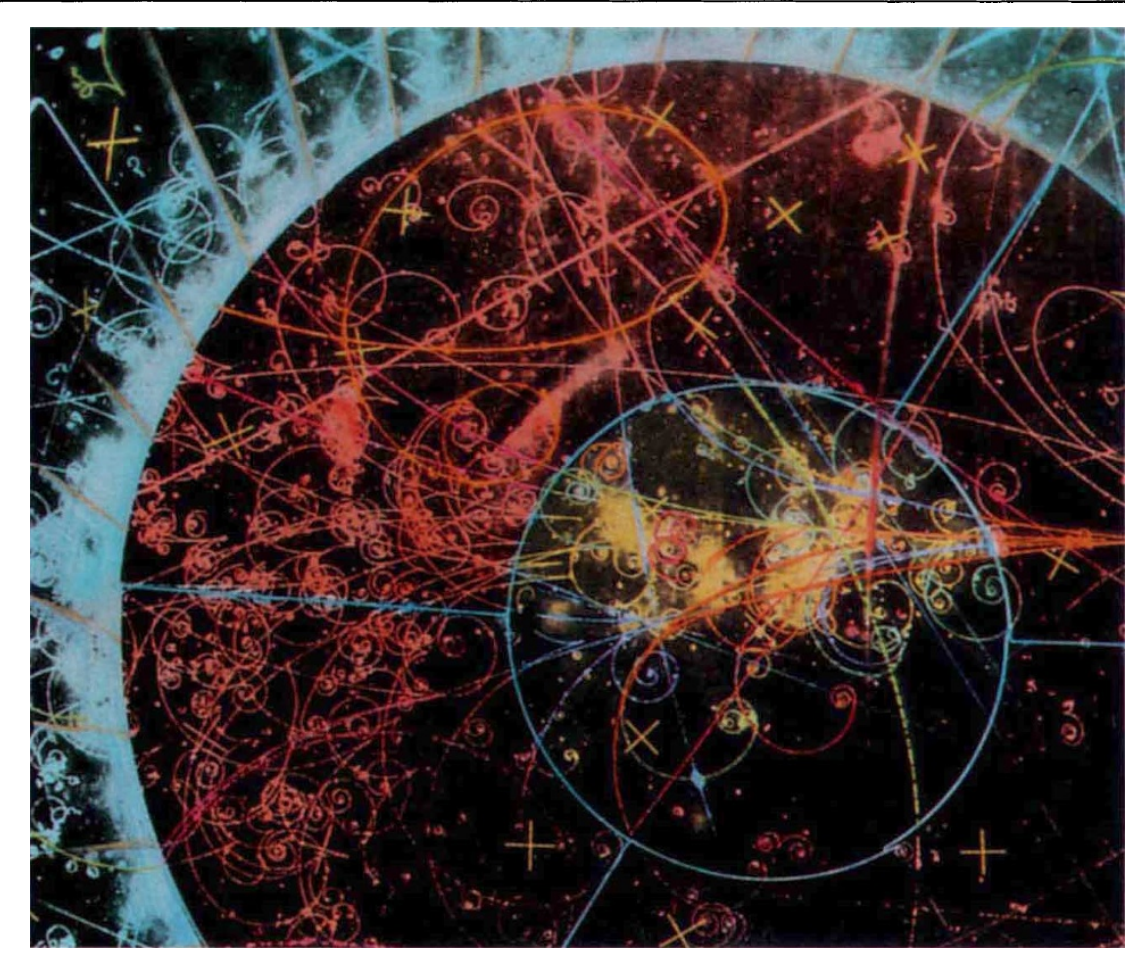

CoLLISION between a high-energy neutrino and the nucleus of a hydrogen atom. Taken from the cover of the paperback edition of From Quarks to the Cosmos: Tools of Discovery by Leon M. Lederman and David N. Schramm. The authors cover exciting new developments in the seven years since the book was first published such as the discovery of the top quark in 1995 and the results of the Cosmic Background Explorer satellite in the early 1990s. They also lament the demise of the Superconducting Supercollider project, but remain optimistic about the completion of CERN's proposed Large Hadron Collider. W. H. Freeman/Scientific American Library, \$19.95, £14.95. 